\title{
Rural Resident Consumption in New-type Urbanization Process in Sichuan Province
}

\author{
Miao WEl ${ }^{1,2, *}$ \\ ${ }^{1}$ School of Economics, Sichuan University, China \\ ${ }^{2}$ College of Tourism, Economics and Management, Chengdu University, China \\ ${ }^{*}$ Corresponding author
}

Keywords: New-type urbanization, Consumption demand, Rural consumption.

\begin{abstract}
In the past 30 years, although the impetus of economy growth in Sichuan Province mainly comes from investment, the contribution of consumption is slight. Consumption rate, especially rural household consumption rate keeps falling. Urbanization is primary method to expand domestic demand. The development of urbanization does not only drive rural household consumption, but also change the pattern of rural consumption, especially in subsistence consumption and expansible consumption. The new-type urbanization emphasizes the overall development of urban and rural places, and emphasizes the population urbanization, which will essentially accelerate the rural household consumption. It is feasible in Sichuan Province to accelerate new-type urbanization to promote the rural consumption so as to expand domestic demand. It can essentially increase the income of rural residents, propel the modernization of the infrastructure in countryside, and level up the consumption so as to create consumption demand.
\end{abstract}

\section{Introduction of Rural Residential Consumption in Sichuan Province}

Consumption demand is the ultimate demand among three major demands, and it is the ultimate goal of social economic production. Only economic development driven by consumption demand is a sustainable economic development mode. However, for a very long time, the economic development in Sichuan Province has been excessively dependent on the investment rather than consumption. The insufficient demand of rural residents is the main cause of the persistent sluggish consumption demand, which has also become the bottleneck of the sustainable economic development in Sichuan Province.

\section{The Overall Level is not High}

Since the reform and opening up, the rural economic development in Sichuan Province has made tremendous progress, and the people's living standards have been greatly improved. However, the rural residents' consumption level still has a yawning gap to that of the developed eastern provinces. As shown in Table 1, the consumption level of rural residents in Sichuan Province is relatively low. In 2015, per capita consumption expenditure in rural areas in Sichuan Province was 9,250.65 RMB, ranking 11th among 31 provinces, municipalities and autonomous regions in China. It's just slightly over the national average, and compared with some developed provinces, the gap is still quite large.

Table 1. Consumption expenditure of rural residents in 2015 (Unit: RMB/ person)

\begin{tabular}{|c|c|c|c|c|c|c|c|}
\hline Nation & Sichuan & Beijing & Shanghai & Tianjin & Zhejiang & Jiangsu & Guangdong \\
\hline 9223 & 9250.65 & 15811.22 & 16152.29 & 14739.44 & 16107.72 & 12882.55 & 11103.03 \\
\hline
\end{tabular}

\section{Rural Resident Consumption Rate Decreased Year by Year}

Sichuan Province is a traditional agricultural province. The rural population accounts for about $80 \%$ of the province's total population, almost $20 \%$ higher than the national average of $60.91 \%$. After 30 years of rapid economic growth, the GDP of Sichuan Province in 2015 will reach 3 trillion yuan. 
However, despite the ongoing economic growth in Sichuan Province, the consumption rate continued to decline, and the contribution of consumption to economic growth was also not optimistic. In 2000, the consumption rate was $64.8 \%$, slumped to $52.5 \%$ in 2015 . In comparison, the investment rate increased from $38.7 \%$ in 2000 to $49.3 \%$ in 2015 . Table 2 shows the composition and trends of consumption demand in Sichuan Province from 2000 to 2015. The statistical figures infer that the decline in the consumption rate is mainly caused by the drop of consumption rate. From 2000 to 2015, the government consumption rate remained stable while the rate of household consumption dropped significantly. The rate of household consumption slumped from $51.5 \%$ in 2000 to $40.2 \%$ in 2015 . This sharp decrease in the household consumption rate is mainly caused by the decline of rural resident consumption rate. Because from 2000 to 2015, the urban resident consumption rate just dropped slightly, from $27.13 \%$ to $25.71 \%$, but the rate of rural residents' consumption slumped from $24.33 \%$ down to $14.47 \%$.

Table 2. Composition and trends of consumption demand in Sichuan Province

\begin{tabular}{|c|c|c|c|c|c|c|c|}
\hline Year & GDP & Government & Urban & Rural & Government rate & Urban rate & Rural rate \\
\hline 2000 & 3928.20 & 523.47 & 1065.82 & 955.84 & $13.33 \%$ & $27.13 \%$ & $24.33 \%$ \\
\hline 2001 & 4293.49 & 616.80 & 1207.03 & 954.93 & $14.37 \%$ & $28.11 \%$ & $22.24 \%$ \\
\hline 2002 & 4725.01 & 676.51 & 1341.90 & 995.86 & $14.32 \%$ & $28.40 \%$ & $21.08 \%$ \\
\hline 2003 & 5333.09 & 751.17 & 1509.34 & 1069.96 & $14.09 \%$ & $28.30 \%$ & $20.06 \%$ \\
\hline 2004 & 6379.63 & 851.30 & 1757.18 & 1197.16 & $13.34 \%$ & $27.54 \%$ & $18.77 \%$ \\
\hline 2005 & 7385.10 & 901.22 & 2038.19 & 1328.28 & $12.20 \%$ & $27.60 \%$ & $17.99 \%$ \\
\hline 2006 & 8690.24 & 1138.06 & 2288.86 & 1397.96 & $13.10 \%$ & $26.34 \%$ & $16.09 \%$ \\
\hline 2007 & 10562.39 & 1386.35 & 2722.01 & 1563.20 & $13.13 \%$ & $25.77 \%$ & $14.80 \%$ \\
\hline 2008 & 12601.23 & 1602.30 & 3226.54 & 1711.33 & $12.72 \%$ & $25.60 \%$ & $13.58 \%$ \\
\hline 2009 & 14151.28 & 1611.20 & 3634.20 & 1967.10 & $11.39 \%$ & $25.68 \%$ & $13.90 \%$ \\
\hline 2010 & 17185.48 & 1971.00 & 4305.38 & 2333.15 & $11.47 \%$ & $25.05 \%$ & $13.58 \%$ \\
\hline 2011 & 21026.68 & 2456.90 & 5175.70 & 2791.80 & $11.68 \%$ & $24.61 \%$ & $13.28 \%$ \\
\hline 2012 & 23872.80 & 2831.40 & 5839.60 & 3255.70 & $11.86 \%$ & $24.46 \%$ & $13.64 \%$ \\
\hline 2013 & 26260.77 & 3120.61 & 6502.92 & 3599.55 & $11.88 \%$ & $24.76 \%$ & $13.71 \%$ \\
\hline 2014 & 28536.66 & 3355.74 & 7156.10 & 4018.10 & $11.76 \%$ & $25.08 \%$ & $14.08 \%$ \\
\hline 2015 & 30053.10 & 3701.52 & 7725.20 & 4348.24 & $12.32 \%$ & $25.71 \%$ & $14.47 \%$ \\
\hline
\end{tabular}

\section{Large Gap between Urban and Rural Residents in Consumption Expenditure}

The widening economic gap between urban and rural areas is a national issue, and it is particularly serious in Sichuan Province. Since the reform and opening up, the economy of Sichuan Province has kept a good momentum of development. People's material and cultural living standards have been greatly improved. However, compared with the living standards of urban residents, the gap is still large (see table 2). This situation does not only reflect the inequality of national income, but also demonstrate a gradient space for opening up rural markets.

Table 3. Comparison of per capita consumption expenditure of urban and rural residents, 2005-2013

\begin{tabular}{|c|c|c|c|}
\hline Years & Rural Residents & Urban residents & Urban-Rural Ratio (Rural = 1) \\
\hline 2005 & 2274.2 & 6891.3 & 3.030208425 \\
\hline 2010 & 3896.7 & 12105.0 & 3.106474709 \\
\hline 2012 & 5366.7 & 15050.0 & 2.804330408 \\
\hline 2013 & 6127.0 & 16313.0 & 2.662477558 \\
\hline
\end{tabular}

\section{The Influence of New-type Urbanization on Consumption of Rural Residents}

"New-type urbanization" is the new engine of Chinese economy, which will provide medium and long term impetus for China's economic growth. Essentially, the urbanization is a process which underpins the industrial structure adjustment and the ongoing improvement of consumption level. In the urbanization process, city civilization will gradually expand and spread so that the overall quality of residents flowing into the city can be improved. At the same time, living conditions, lifestyle and 
quality of life can also be improved. In the context of globalization, the urbanization of Sichuan Province has entered into a new era of development and transformation. The new-type urbanization is a rational urbanization to meet the objective requirements of Sichuan Province's economic, social and natural development. It is also an active urbanization in line with China's national conditions and future.

\section{The Urbanization Rate and the Trend of Rural Residents' Consumption Change in Sichuan Province}

This paper selects the data of Sichuan Province from 2005 to 2012 to investigate the relationship among the urbanization rate, the consumption expenditure and the consumption structure of rural residents. The consumption structure is demonstrated by the proportion of each expenditure to the total expenditure per capita. As can be seen from table 4, in recent years, with the continuous improvement of urbanization rate in Sichuan Province, the per capita consumption expenditure of rural residents is also increasing at the same time, from 2247.2 yuan per capita in 2005 to 5366.7 yuan per capita in 2012, with an average annual growth rate of $8.3 \%$. The changes in the consumption structure show the following trends:

(1) The proportion of food expenditure (Engel coefficient) and living expenses accounted for the larger proportion of the consumption expenditure, and the proportion of food expenditure with the urbanization rate showed a reverse trend. 2005-2012, the food consumption of rural residents living consumption accounted for the proportion (Engel coefficient) maintained at the $46.85 \%-54.72 \%$ and the rural residents for food consumption expenditure accounted for the proportion of consumption expenditure decreased from $54.72 \%$ in 2005 to $46.85 \%$ in 2012 . The proportion of living expenditure is on the rise, rising from $10.29 \%$ in 2005 to $14.67 \%$ in 2012.

(2) The proportion of living and consumption of clothing, household equipment and service expenditure is small, and it is slowly rising. In the past 2005-2012 years, the proportion of clothing consumption of rural residents to total consumption remained at $5.12 \%-6.31 \%$, and household appliances and services accounted for $4.49 \%-6.21 \%$. These two items are on the rise, but the growth is small.

(3) The proportion of entertainment expenditure in consumer spending is large in 2005, but in recent years, it has shown a clear downward trend and down to $6.14 \%$ in 2012 .

(4) The consumption of transportation, communication and medical expenditure maintained a fast growth momentum. In 2005-2012, the province's rural residential spending on transport or communication and medical kept an average annual growth rate of $24.36 \%$ and $34.98 \%$ respectively, higher than that of rural residential living consumption expenditure growth; effectively promote the proportion of traffic and medical expenditure in consumption expenditure [1].

\section{Expansion of Rural Consumption Demand with New-type Urbanization}

New-type urbanization redefines and makes new planning for the development of urbanization. It is no longer just pursuing the upgrading of urbanization expansion, but attaching more importance to the development quality of urbanization. New-type urbanization is characterized by co-ordination of urban and rural areas, integration of urban and rural areas, interaction between industry and urbanization, combination of economization and intensivism, as well as livable environment and harmonious development. And it emphasizes on the coordinated development and mutual promotion of large/medium/small-sized cities, small towns and new rural communities. Therefore, this new concept of urbanization can facilitate the promotion of rural consumption demand, and accelerate the upgrading of the consumption structure. Specifically, the benefits of new-type urbanization brought to the promotion of rural resident consumption mainly include the following aspects:

(1) New-type urbanization can increase the income of rural residents

Consumption is a functional result of income. The expansion of consumption demand ultimately depends on the increase of income. Increasing farmers' income is the key to raising farmers' 
purchasing power and expanding rural consumption. With the development of new-type urbanization, on the one hand, a large number of rural surplus labor will transfer to towns, and the population directly engaged in agriculture will be greatly reduced, and the area of cultivated land will relatively increase. This is conducive to intensive land management, so as to promote agricultural modernization faster and better, thereby improving agricultural labor productivity and increasing farmers' income [2]. On the other hand, the transfer of surplus rural labor force into cities and towns will lead to a higher income level. With the improvement of the income level of rural residents, the consumption demand will also increase.

(2) New-type urbanization can promote the upgrading of urban and rural residents' consumption structure

The upgrading of consumption structure is an important factor to increase consumption demand. With the advancement of urbanization, the improvement of residents' income level and the improvement of the consumption environment will inevitably expand the consumption fields and make significant changes in the consumption structure. Due to the consuming demonstration effects among city residents, those products and services unseen in rural areas will continually be known by rural residents, and will stimulate their desires of consuming. The result is consumption mode in small towns and rural areas begins to get close to or converge to that of cities. Rural consumers actively imitate urban consumers, and their original consumption concept, the consumption patterns and consuming preferences are constantly updated. The demands of service consumption such as health care, transportation and communication, education and culture, recreational services and housing will be increasingly stimulated. As a consequence, the development of consumer credit service becomes more vigorous, hence promote the increase of consumption demand and accelerate the upgrading of consumption structure. We can conclude that the development of urbanization can not only promote the development of public services such as education, health-care, social security, employment, but also can promote the development of consumer services such as trade, catering and tourism, as well as production services like finance, insurance, logistics. The development of urbanization can provide endless business opportunities for the development of the service sector [3].

(3) New-type urbanization is beneficial to stabilizing the income expectation of rural residents

On the one hand, under the guidance of the concept of urban and rural co-ordination development, new-type urbanization emphasizes the balanced and equal development of urban and rural areas, and effectively promotes the integration and cohesion of urban and rural social security system. The social security system of urban and rural integration can narrow the differences between urban and rural social security system, improve the rural residents' social security efforts, reducing rural residents of income menace from the rear, stable expectations, enhancing rural consumer confidence.

On the other hand, the new-type urbanization focus on solving the problem of semi urban, to allow the transfer of rural labor force to obtain citizenship, the rural surplus labor force into the city life in the urban employment, household registration, change from rural household to urban areas, and urban residents will be able to enjoy the same, originally as rural residents to enjoy or even but to enjoy the discount rate is far below the level of the city all kinds of social insurance and housing welfare, education and other social welfare benefits [4]. At the same time, the medical level and education level of the city are relatively high, which provides guarantee for the health of the people of the city and the education of children. Change transfer for city residents of the rural labor force more perfect in the urban social security system, the risk of future expected decline, precautionary savings reduced, therefore, the income will be used for current consumption.

(4) The new-type urbanization is beneficial to the improvement of the rural consumption environment

New-type urbanization emphasizes on the overall development of urban and rural areas, i.e. we do not only accelerate the construction of urban areas but also pay attention to the construction of rural areas. New-type urbanization process will pay attention to the construction and improvement of rural infrastructure, including rural roads, water, electricity, communications, health, culture and 
entertainment infrastructure, and create conditions for the entry of consumer durable goods, household appliances into rural areas [5]. In this way, more modern consumer goods will enter the farmers' families, raise the level of farmers' living consumption, and will also bring new spring to the consumption of household appliances.

(5) New-type urbanization can improve the concentration of consumption

The diversity of consumers' demands for products and manufacturers' pursuit of economies of scale will encourage consumers and manufacturers to accumulate in urban areas where agglomeration effects can be generated, resulting in an increasing consumption agglomeration effect [6]. In areas between urban and rural areas, it will generate a similar effect, with the increasing urban population and rising of income levels, urban demands, including demand for agricultural products, will be expanded [7]. The increase of demand for agricultural products is bound to increase the income of rural residents and thus expand consumption, so as to promote the further development of city industry and service sector. Besides, it can absorb more rural people into cities and towns, thus forming a virtuous cycle of expansion and cumulative effect of consumer demand.

\section{Measures of Accelerating the Construction of New-type Urbanization}

(1) Pay attention to the co-ordination of urban and rural development and form the economic integration of urban and rural areas

The construction of new-type urbanization is one of the main driving forces to stimulate consumption demand in rural areas. Accelerating the construction of new-type urbanization in Sichuan Province is guided by the idea of coordinating urban and rural development based on industrial support and accelerating the reform of household registration system and the reform of rural land system.

(2) Strengthen industrial support for new-type urbanization and create stable employment opportunities

The integration of urban and rural areas is the core of the new-type urbanization. It is an urban and rural system which emphasizes the urban and rural integration and the compatibility between urban and rural areas. Farmland, woodland, mountains and rivers are the carrier of modern agriculture, and in the meantime, they are the ecological function areas of the city. We should change the thinking ways to tilt resources into large and medium-sized city, and instead, should try to focus on the concept of fairness and efficiency of urbanization, co-ordinate urban and rural development, implement the integration of planning, infrastructure construction, public service provision in urban and rural areas. Its target is to narrow the gap between urban and rural areas, to achieve co-prosperity of urban and rural areas, and improve rural residents' consumption ability.

The new-type urbanization can't be separated from the support of the industry. It is necessary to promote the industrial development to enhance the ability of cities and towns to create jobs for the surplus agricultural labor force and to strengthen the industrial support for urbanization. First, it is necessary to change the mode of industrial development, adjust the internal structure of industries, upgrade traditional industries and foster new industries. Second, efforts should be made to increase the proportion of urban service industries and create a favorable policy and institutional environment for the development of service industries. Third, we should rationally plan the infrastructure construction such as transportation, communications and networks to create favorable conditions for the development of urban industries. The fourth is to build a vocational training system and entrepreneurial support system for urban population, promote employment through industrial development, and improve the employment quality of agricultural transferred labor so that they can be better integrated into cities. The new-type urbanization with industrial support can provide stable employment opportunities for rural migrant workers and provide them with more stable income. This is also the basis for the expansion of consumer demand.

(3) Actively promote the reform of household registration system 
First, we should implement the elimination of the dual household registration system and its related pension, health care, education, housing, social security and other institutional obstacles against the transformation of the peasants into the citizenization. Second, we should completely solve the transfer of rural labor force, and get rid of their policy worries. This will contribute to the enhancement of rural labor transfer and consumer confidence, so as to enhance the consumer demand.

(4) Speeding up the reform of rural land system

New-type urbanization should aim at the increase of rural residential per capita resources possession, by promoting the innovation of land system, promote the legal and orderly transfer of land, in-depth implementation of land transfer, leasing, price of shares and other combined means, to use land resources reasonably. The key is to promote the scale management of the land, increase the income of the agriculture by increasing the efficiency of the land labor, and increase the income of the farmers, so as to expand the consumption of the rural residents.

(5) Strengthen the guide of consumption, change the concept of consumption and consumption patterns

The construction process of the new-type urbanization is not only the process of farmers' integration into the city, but also the process of urban civilization's radiation and extension to the countryside. The appropriate consumption guide through the transfer of rural labor, gradually change the concept of consumption, promote consumption patterns from current self-accumulation mode to credit support mode. With the support of policy, it guides rural financial institutions to tilt the consumer credit of rural residents, implement subsidies for rural consumer credit, so as to encourage consumption demand of rural residents and realize the expansion of rural consumer market.

\section{Conclusion}

In this paper, we have analyzed current situation of rural resident consumption in Sichuan Province. The insufficient demand of rural residents is the main cause of the persistent sluggish consumption demand. We also find out that the new-type urbanization is a rational urbanization to meet the objective requirements of Sichuan Province's economic, social and natural development. And finally, we provide the measures for accelerating the construction of new-type urbanization in Sichuan Province and promoting the rural consumption.

\section{Acknowledgement}

This research was financially supported by Project of Sichuan Provincial Department of Education, No.16SB0333.

\section{References}

[1] X. Wang, Changes of rural resident consumption — based on data in Sichuan [J]. Rural Economy, 2014(8): 59-61.

[2] C. Zhu. Suggestions for increasing persistent income of rural resident and expanding rural consumption [J]. Consume Guide, 2009, (11): 48.

[3] H. Zhang, D. Wang, The new-type urbanization, the industrial structure adjustment and the rural labor "reemployment"“J]. China Soft Science, 2016(6): 136-142

[4] T. Wang, M. Zhu, The new-type urbanization and rural resident income increasement: mechanism, method and solution [J]. Fujian forum, 2014(11): 30-37

[5] L. Yan, Therotical logic and solution selection of urbanization improvement [J]. Study and Practice, 2014(2): 17-26. 
[6] Y. Chen, Suggestions for improving the development of new-type urbanization in Sichuan [J]. Reform of the Economic System, 2010(6): 133-137.

[7] S. Xie. Expanding the rural resident consumption in construction of new-type urbanization [J]. Macroeconomic Management, 2014(11): 74-77. 